\title{
Parallel Standard Cell Placement on a Cluster of Workstations*
}

\author{
Faris H. Khundakjie, Patrick H. Madden \\ Nael B. Abu-Ghazaleh and Mehmet Can Yildiz \\ State University of New York at Binghamton \\ Computer Science Department \\ nael@cs.binghamton.edu
}

\begin{abstract}
In this paper we report experiences on a parallel implementation of a standard cell placement algorithm on a cluster of Myrinet connected PCs. The implementation is based on a recently developed placement tool (Feng Shui) that extends recursive bisection placement to incorporate global aspects of the design using an efficient optimization called iterative deletion. Contrary to previous attempts at parallelizing placement algorithms, initial experimental results show significant performance improvement with small reduction in the placement quality. Furthermore, the reduction in the placement quality does not increase with the number of processors.
\end{abstract}

\section{Introduction}

With advances in VLSI fabrication technology, the size of circuits of interest is continuously expanding. Physical design automation tools are needed to aid in design and layout of such circuits. However, the size of the circuits presents a similar challenge to the design automation tools: they must be able to provide good quality layouts with acceptable run times. In this paper, we consider VLSI standard cell placement - an important and difficult problem in physical design automation. The placement impacts circuit areas and wire delays profoundly; a poor placement may prevent a circuit from operating at an acceptable speed, or make it too large for the available real estate. Furthermore, if the placement algorithm has high complexity, we will be unable to obtain a placement in an acceptable time, forcing a sacrifice in the placement quality (performance, area) to achieve faster placement time.

Parallel processing offers the promise of increasing the performance and capacity of placement tools, enabling them to provide better solutions in faster times. The emergence and commercial success of clustering technologies is perhaps the most exciting development yet in the field

* This research was supported in part by NSF under awards CCR9988222 and EIA-991099 of parallel processing: it allows scalable cost-effective parallel processing machines to be built $[1,3,27]$. Clusters approach the performance of custom parallel machines by using high-performance Local/System area networking technologies and standards (such as Myrinet [5], SCI [15] and others $[4,34,35])$ and low-overhead userlevel space communication protocols (such as the Basic Interface for Parallelism (BIP) [13], Illinois Fast Messages (IFM) [26] and others [9, 25]). Because they use commodity components, clusters are affordable, scalable and easy to build [30].

This paper presents experiences in parallelizing a stateof-the-art placement tool on a cluster of workstations. The algorithm uses a standard iterative bisection approach augmented with an iterative deletion step that incorporates global aspects of the design in addition to local optimization obtained through iterative bisection. The contributions of the paper are: (i) the initial sequential algorithm is state-of-the-art from a run-time complexity and solution quality perspectives; (ii) the parallel implementation provides significant speedup while maintaining high quality solutions regardless of the number of processors used. In contrast, other parallel implementations generally suffer significant degradation in quality; (iii) most published solutions are specific to an architecture and are not directly portable to a cluster environment. To our knowledge, this is the first study that investigates this important problem on a modern cluster environment; and (iv) we investigate several algorithmic optimizations as well as system-level tradeoffs in the implementation. This includes performance comparison of a Myrinet network [5] running the BIP message passing library relative to using $100 \mathrm{Mbit} / \mathrm{sec}$ switched Ethernet for communication.

The remainder of this paper is organized as follows. Section 2 the placement problem and related work. Section 3 discusses the sequential placement algorithm in more detail. Section 4 presents the details of the parallel implementation. Section 5 presents the experimental setup and results. Finally, Section 6 presents some concluding remarks. 


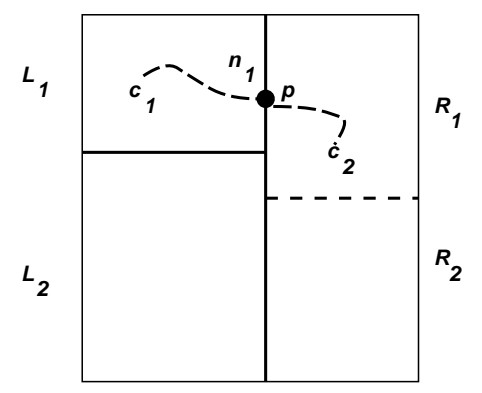

Figure 1: Terminal propagation as performed by Dunlop and Kernighan. When recursively partitioning a netlist, we can insert dummy terminals to influence the partitioner. If a net spans more than one region, the location of dummy terminals can improve the placement quality.

\section{Overview and Related Work}

We consider the problem of standard cell placement: the objective is to place rectilinear circuit elements (cells) into one or more horizontal rows, minimizing total wire length. There are a number of established approaches to the placement problem. For a comprehensive survey, the reader is referred to the following paper [31]. Force Directed or LP based approaches repeatedly solve systems of equations, determining cell locations iteratively (for example, [11]). This approach is popular in commercial placement tools. Simulated Annealing approaches obtain cell placements by swapping positions of cells randomly, guided by a probabilistic acceptance function. A number of current commercial placement engines (e.g., TimberWolf[33]) use this approach; efficient cost estimates allow the consideration of large numbers of intermediate states. Partitioning based approaches determine cell locations by recursively dividing an initial area (region) with successive bisections or quadrisections. This approach has become more attractive recently; advances in partitioning research have provided a number of fast algorithms which produce extremely good results. This is the approach used in this paper.

Breuer[6] utilized repeated graph bisections to obtain a circuit placement.The bisections divide the circuit netlist into a hierarchy of cells, with the resulting hierarchy roughly mapping into a rectilinear grid. Dunlop and Kernighan[10] extended this approach, through the use of an improved partitioning method[18].

Dunlop and Kernighan [10] also introduce terminal propagation. When partitioning a region, we can expect a number of connections to be required to cells or pads outside of the region. Terminal propagation provides a simple method to insert fixed "dummy" vertices, so that the partitioning considers these external connections (Figure 1).
With terminal propagation, the partitionings of regions become interdependent; if we begin with two regions, $L$ and $R$, and partition $L$ first, this impacts the optimal solution for $R$. Partitioning $R$ first might result in a different solution, and neither of these might be globally optimal, even if the individual partitionings were. To address the order dependence of the partitioning, both [32] and [14] employ repeated partitioning at each level. We might wish to partition $L$, followed by $R$, and then partition $L$ a second time. Repeated partitionings do not, however, change a local optimization process into a global one.

Because of the computational complexity of placement, there has been significant interest in parallelizing placement algorithms since the 1980's (see for example $[8,16,19,20,21,22,24]$; a good summary is available here [2]). These studies have experimented with parallelizing most of the placement algorithms on a variety of parallel architectures. Most of these studies are quite old and are specific to the architectures that were used to test them; it is difficult to compare performance directly with them. In addition, the objective functions that the different studies optimize are generally different. Furthermore, research in placement suffers from the lack of uniform metrics for reporting the results - wire lengths are reported, but the results can vary by a multiplicative factor depending on the underlying assumptions (such as cell spacing and the method of measuring wire length). This makes it difficult to fairly compare the algorithms, even when the same models are used. However, while direct comparison is difficult, comparison of relative measures between the sequential and parallel versions of each algorithm (such as speedup, and quality degradation) are still possible. In the following paragraph we will overview some of the most recent of these works. In Section 5 they are also reviewed as we compare our results to them.

Banerjee's research group has done the most extensive work in the area of parallel placement algorithms. For example, within the ProperPLACE CAD tool they discuss a parallel placement algorithm based on simulated annealing [19]. In contrast to other parallel placement implementations, they work with an abstract parallel machine model allowing the implementation to be directly ported across different architectures. They study shared memory and distributed memory implementations. The speedup across different implementations were reported on the ISCAS benchmarks. Koide et al present a parallel implementation of their POPINS timing driven placement tool [21]. They use a bisection approach similar to our own, with non-linear programming used in a second phase optimization. The parallel implementation uses shared memory. In this study, the drop of quality in the parallel implementation was significant (an average of 14\%). 


\section{Algorithm Formulation}

This paper considers parallelizing a placement tool (called Feng Shui [36]) developed by two of the authors. Feng Shi adapts a recently presented partitioning approach for $k$-way partitioning. It differs from that solution in that it uses a technique called iterative deletion [23] to allow some global issues to be captured resulting in improved placement quality at significantly lower cost than $k$-way partitioning. Feng Shui integrates a variant of $k$-way partitioning approach into a traditional framework. The general flow of our placement tool is as shown in Figure 3. When faced with large numbers of regions to bisect, iterative deletion is applied first (to obtain a good quality global solution), followed by repartitioning of regions with a traditional bisection approach (to improve solution quality from a local perspective). The remainder of this section describes these steps in more detail.

\subsection{Bisection}

The framework for the placement tool is a textbook implementation of the approach of Dunlop and Kernighan[10]. The circuit is repeatedly divided by either horizontal or vertical cut lines. A recent multi-level clustering based partitioning algorithm hMetis[17], version 1.5.3 is used. At each partitioning, we attempt to obtain a nearly exact bisection if cutting vertically. If the cut line is horizontal (splitting a number of rows), the rows are split as evenly as possible. If the region being bisected contains an odd number of standard cell rows, fixed and weighted dummy vertices are added to allow a nearly exact bisection to be mapped into the available space. The partitioning objective is min-cut; the hMetis partitioner attempts to minimize the number of cut hyperedges.

Recursive bisection divides placement regions into progressively smaller areas, ultimately assigning each cell to a single row, but possibly having several cells remaining within a region. To establish positions for each cell, the cells are ordered by region location within each row; the cells are packed together without spaces or overlap. The positions of cells which were within the same region will be arbitrary at this point; they were not ordered by the partitioning process. To optimize these positions, we apply branch-and-bound reordering to modify the positions of a small set of consecutive cells in a single row.

Feng Shui allows the specification of a "window size," controlling the number of cells involved in any branchand-bound optimization. This window passes over each cell row (in order), traveling along each row at steps of half the window size. At each step, the optimal order for cells found under the window is determined. The number of passes over the placement, and the size of the win- dow, are both parameters which can be controlled by the user. In practice, we find that window sizes of 6 to 8 cells, and 4 passes of improvement, are sufficient for good overall performance. Increasing the window size impacts run times substantially (the complexity of the branch-andbound procedure is $O(w !)$ worst case, where $w$ is the size of the window). In [7], a number of ways to implement branch-and-bound reorderings efficiently were explored.

\section{$3.2 k$-Way Partitioning}

The focus of our work has been on the global aspects of the placement problem. With partitioning, we can optimize the number of edges cut within a region effectively, but have no way of knowing if this local optimization is appropriate from a global perspective. Similarly, our branch-and-bound reordering is also a local optimization.

A careful examination of placement by recursive bisection reveals a number of instances where global objectives may be lost. The example in Figure 3 shows a simple case where local optimization is insufficient; there are four regions to bisect, each with two cells. If the problem is approached as a series of independent bisections, a number of configurations which are both stable and suboptimal can be encountered. The sub-optimality of the global solution is not related to the quality of the bisections of each region; simply improving the bisection algorithm will not improve the global configuration.

As we progress through the placement process, the number of regions increases, doubling repeatedly. If there are $k$ regions that are to be split (obtaining $2 k$ new regions), the traditional approach is iterative, bisecting a single region at a time. Instead, Feng Shui attempts bisection of all regions at the same time, obtaining a solution that is of good quality globally. The method used to perform this massive bisection is based on partitioning by iterative deletion[23].

\subsection{New Formulation - Iterative Deletion}

To capture global objectives effectively, a variant of multiway partitioning is used (rather than as a series of bipartitions). We partition all regions simultaneously, with the intermediate state of each region influencing the others. We are concerned with partitioning very large numbers of regions, and our cost objective is wire length rather than min-cut. The problem we consider is given a set of regions (with physical constraints) and a set of elements mapped to these regions, bisect all regions to minimize the resulting bounding-box wire length. Solution of this problem optimizes the circuit from a global perspective. Multi-way partitioning has proven quite challenging[29]; for traditional objectives such as min-cut, the greatest success has been obtained with recursive partitioning. 


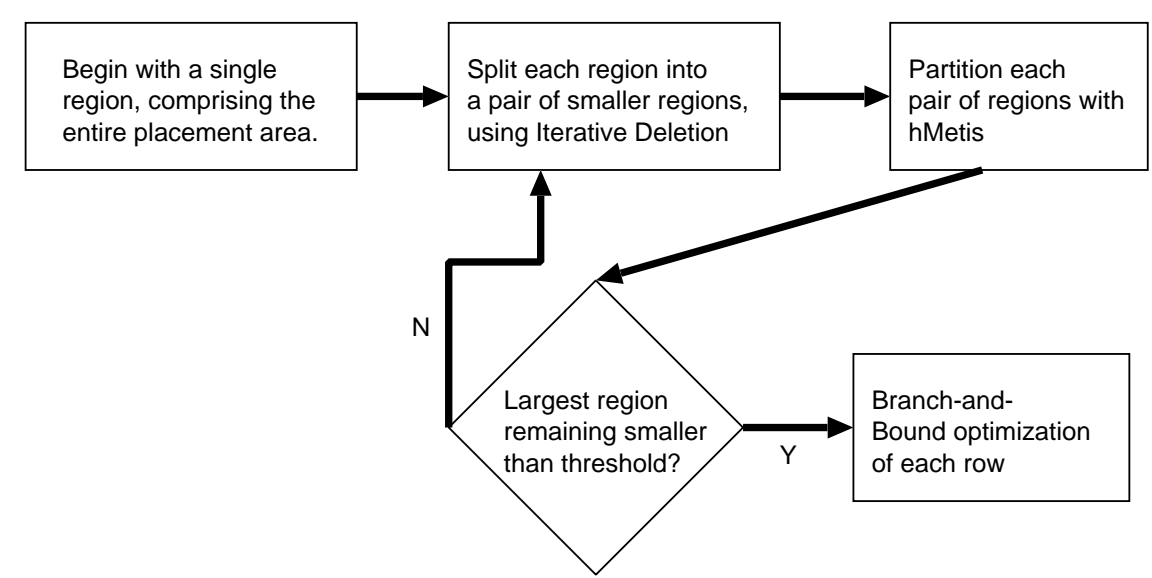

Figure 2: Flowchart of the placement approach. The partitioning and branch-and-bound improvement steps are well known. New to the approach is the pre-processing performed by iterative deletion.
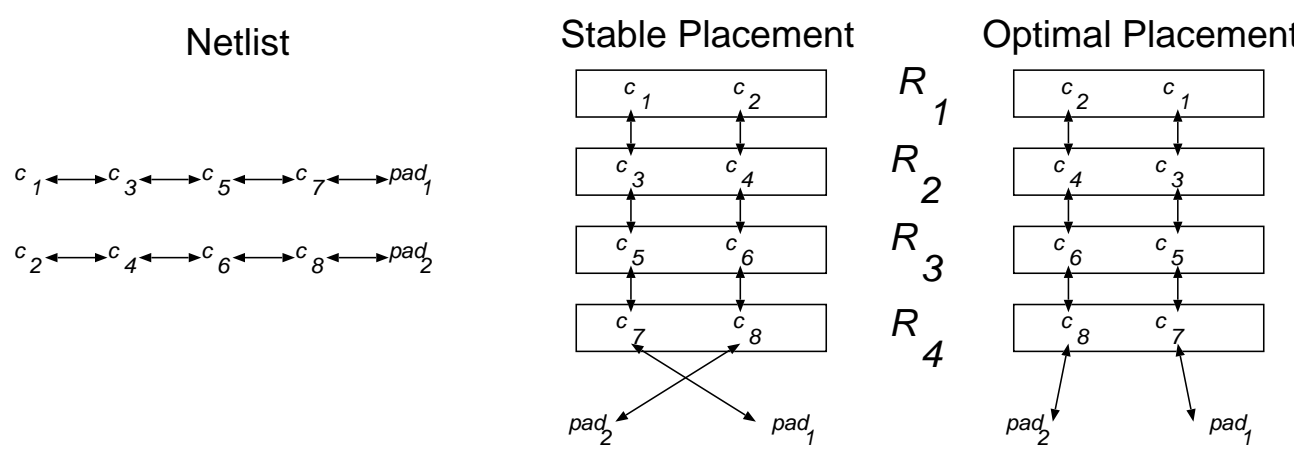

Figure 3: Given the netlist above, we may assign pairs of cells to the regions shown. If we determine the ordering in region $R_{1}$ first, we arrive at a stable but suboptimal solution.

In [23], hypergraph partitioning was considered. A new method based on iterative deletion was presented; in this approach, vertices are duplicated, with one instance of each vertex being assigned to a partition. Redundant elements are removed one at a time until no duplicates remain. While the approach was relatively simple, it proved effective in some areas where traditional methods had difficulty. For bipartitioning, cut sizes from a single lineartime pass were comparable to many passes of a traditional FM[12] algorithm. Multi-way cut sizes were superior to a direct flat multi-way partitioning algorithm[29]. For problems with a variety of hyperedge weights, a combination of iterative deletion and FM partitioning proved substantially more effective than FM partitioning alone. The approach is computationally attractive: with integer hyperedge weights, it may be implemented in $O(n)$ time.

Our variation of the iterative deletion approach for placement operates in the following manner. Each cell in a region is assigned to both subregions; if there is more than a single instance of a cell, it is considered to be $r e$ - dundant. We repeatedly remove redundant cells from subregions which have high utilization, and select the highest cost cell for removal.

In the existing implementation of the placement engine, the cell cost is evaluated based on the center of mass for the component nets. For each net $n_{i}$, the center of mass for this net is the average $X$ and $Y$ location of the cells which it connects. The cost of any cell $c_{i}$ is the sum of the distances between the cell and the center of mass of each net to which the cell is connected. In this way, a cell which is far from the center of mass of each net to which the cell is connected has high cost. Each region has a number of cells assigned to it, and an available capacity; redundant cells (those introduced by iterative deletion) with high costs are removed from the region which has the highest ratio of cell area to capacity.

Heaps are used to maintain the ordering of cells within any given region and the ordering of regions. In this way, maintenance and cell selection are both at worst $O(\log n)$ for each cell removed. As the number of redun- 
dant elements to be removed in any pass is $n$, each pass is $O(n \log n)$. Region sizes decrease by a factor of 2 with each pass, resulting in a logarithmic number of passes required. Thus, the iterative deletion portion of the algorithm is at worst $O\left(n \log ^{2} n\right)$.

To illustrate the iterative deletion process, we present Figure 4. In this figure, we duplicate cells $c_{1}$, in region $R_{1}$, and cell $c_{2}$ in region $R_{2}$, and assume that a net connects $c_{1}$ to $c_{2}$, and that a second net connects $c_{2}$ to a pad.

The order of cell deletions in this figure is as follows. Note that other cell deletions may be interspersed with the steps; we focus only on these cells to clarify the process.

- The center of mass for the nets connected to cell $c_{2}$ is closest to the pad; we remove the instance of $c_{2}$ which is furthest from this location (as this is the instance which has highest cost).

- The center of mass for nets connected to cell $c_{2}$ is recalculated, and this is propagated to the other cells.

- Net $n_{1}$ now has two instances of $c_{1}$ and one instance of $c_{2}$ connected to it: the center of mass for this net changes, influencing the cost for each instance of $c_{1}$.

- An instance of $c_{1}$ is removed.

\section{Parallel Cell Placement}

The optimized sequential implementation of the placement algorithm discussed above was instrumented to isolate the portions that are most computationally demanding. The bulk of the execution time was spent in partitioning and reordering phases - iterative deletion updates contributed very little overhead after each partitioning pass. The parallel implementation and optimizations to it (both those we have already implemented and ones that are planned) will be discussed in this section. The implementation was performed using MPICH running on top of the Basic Interface for Parallelism (BIP) [28] on a myrinet connected $\mathrm{PC}$ cluster running Linux.

\subsection{Parallel Partitioning}

In order to maintain the global optimization achieved by iterative deletion, parallel processing is restricted to the growing list of regions to be partitioned in each pass. In the first pass the main region is partitioned by a master process into two. In the second pass the resulting two regions are partitioned into four in parallel by the master and a slave and so on until the processor limit is reached. As the list grows, regions above a certain threshold are distributed evenly by the master among all processes. An example is shown in Figure 5.
Initially, we implemented the data distribution using a message per work unit (i.e. region). Thus, the master walks the list and sends a message for each region to the slaves in a round-robin fashion. We optimized this implementation by generating a message per each batch of work units using MPI's vector scatter-gather. Obviously, the latter outperforms the first because it increases the computation granularity by requiring the master to communicate fewer messages with larger size. Load balance is maintained by the aforementioned even distribution of regions and by the fact that the partitioning engine produces balanced region cuts as well.

Because VLSI CAD applications require large memory space, it is necessary to utilize memory efficiently. Therefore, unicast master-to-slave and broadcast communication messages are used to instruct slaves to allocate only the space required for storing and processing their workload in round robin and vector scatter-gather implementations respectively. Such messages incur slight overhead that is negligible for non-trivial placement problems.

An approximate analysis of the parallel partitioning follows. Consider a region of area $\mathrm{R}$ to be partitioned into $\mathrm{N}$ regions just below the threshold. Assuming a linear run time complexity of the partitioning engine and a homogeneous distribution of circuit elements, if it takes time $T_{p}$ to partition $\mathrm{R}$, then partitioning a region of area $\frac{R}{n}$ requires $\frac{T_{p}}{n}$. The progression of region partitioning is modeled by the tree in Figure 5. A sequential implementation performs n steps, with step time $\frac{T_{p}}{n}$, at each pass to partition $\mathrm{n}$ regions into $2 \mathrm{n}$ regions with a total time of $T_{p}$. The number of passes is $\log _{2} \mathrm{~N}$, and so the total amount of partitioning time is $T_{p} \cdot \log _{2} N$. On the other hand, using P processors, the first $\log _{2} P+1$ passes have $n<=P$ and each takes $\frac{T_{p}}{n}$ to complete (i.e. a maximum of one region per processor) while the rest have $n>p$ and each takes ceil(n/p) × $T_{p} / \mathrm{n}$ to complete (i.e. ceil(n/p) regions per processor). The time for the first group of passes is:

$$
\sum_{i=0}^{\log _{2} P} \frac{T_{p}}{2^{i}}
$$

and the total time for the rest is:

$$
\sum_{i=\log _{2} P+1}^{\log _{2} N-1} \frac{T_{p}}{P}=\frac{T_{p}}{P} \cdot\left(\log _{2} N-\log _{2} P-1\right)
$$

For machines with small $\mathrm{P}$, over a wide range of problem size, $\mathrm{N}$ is much larger than $\mathrm{P}$ (i.e. the number of passes with $n<=p$ is much less than those with $n>P$ ), and if $\log _{2} N>>\log _{2} P+1$, then the total time is given by the second formula and is approximated by $\frac{T_{p}}{p} \cdot \log _{2} N$. The best case speedup (sequential/parallel time) is hence linear in the number of processors. 


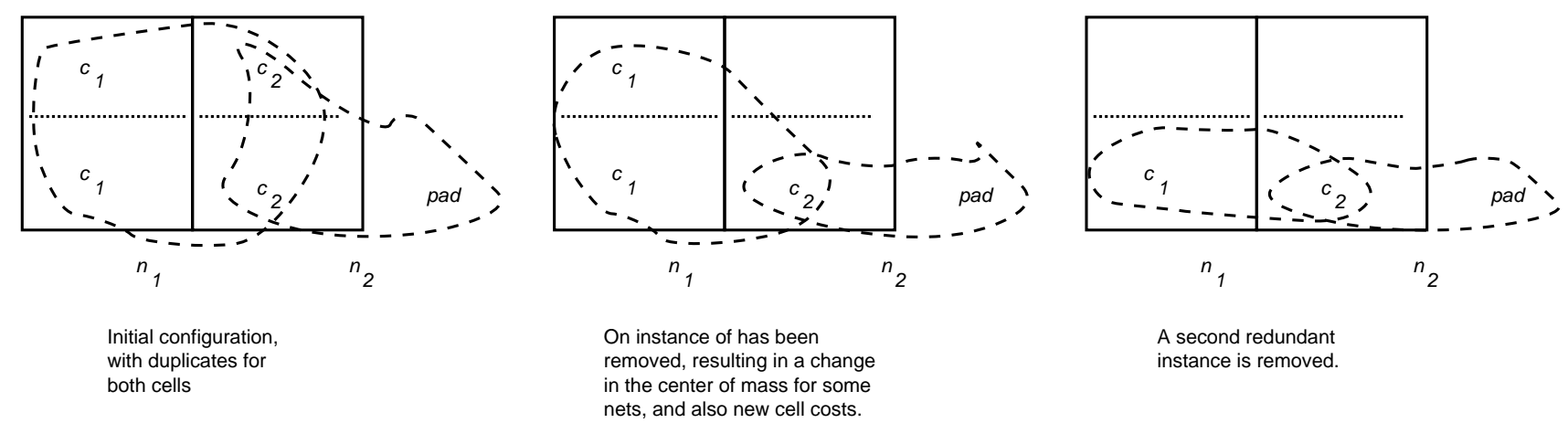

Figure 4: Two instances of each cell in the netlist are generated, and assigned to both subregions of any region. Cell costs are based on the center of mass for the vertices; high cost cells are removed one at a time from any region of the entire placement problem. Partitioning is performed on a global basis, rather than with a pair of subregions at a time.

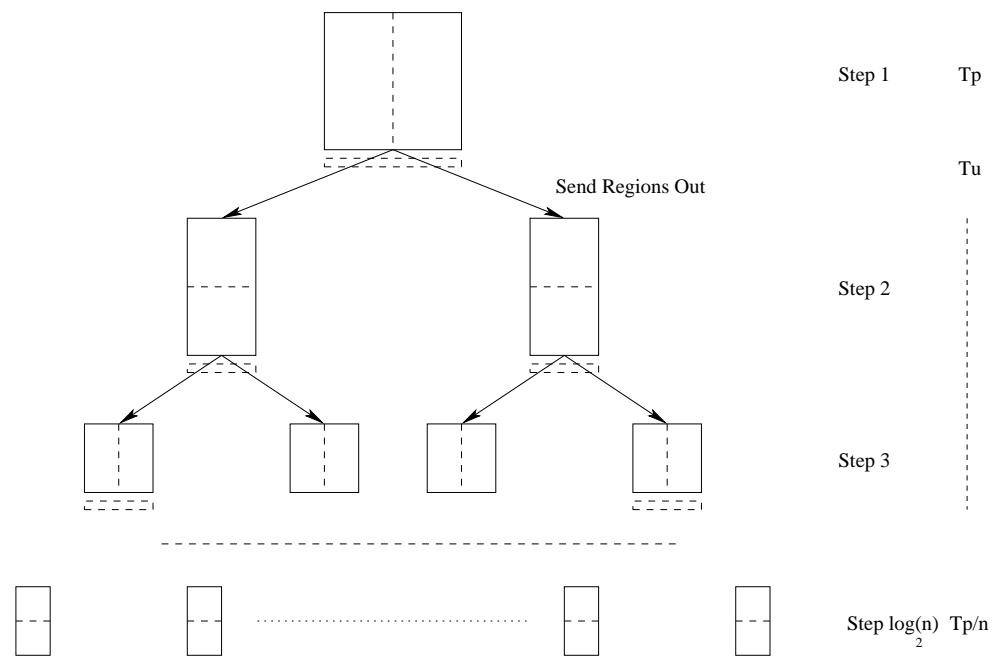

Figure 5: Parallel Partitioning

Cost update and communication times could be incorporated by time $T_{u}$ after each pass. The total of this overhead is the geometric series and is given by:

$$
\sum_{i=0}^{\log _{2} N-1} 2^{i} \cdot T_{u}=T_{u} \cdot \frac{\left(2^{\left(\log _{2} N\right)}-1\right)}{(2-1)}=(N-1) \cdot T_{u}
$$

Since $T_{u}$ is larger in the parallel implementation due to communication time, the actual speedup cannot be expected to be linear.

\subsection{Parallel Reordering}

Branch and bound cell reordering is a second phase optimization that is performed after the placement step to improve the solution quality. A reordering window of a prespecified width in number of cells is slid across the each row in steps of half the window size. All combinations of reorderings of the cells within the current window are considered and the best window kept. Thus, the smallest work unit is the block of consecutive cells defined by the reordering window. The larger the window size the larger the block and the large the number of cells considered for reordering at a time, but the smaller the number of blocks since rows are of fixed length. All cells except half a window's worth at the beginning of each row are considered for reordering twice. To keep this key for quality in the parallel implementation, only non-overlapping blocks are reordered in parallel. Thus, each row undergoes two passes of reordering where a window now moves at steps of full size. The first pass considers even blocks only and the second considers odd blocks only or vice versa.

The same principles for partitioning load balance and communication are followed here; blocks are distributed 

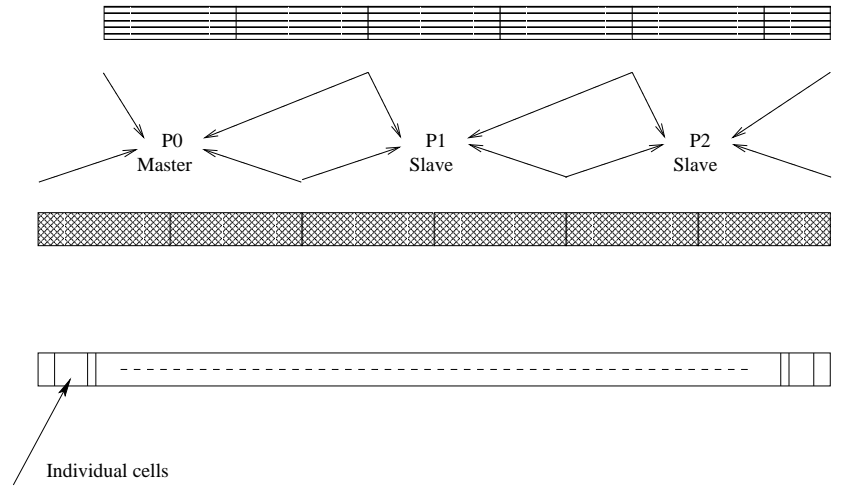

Data Set

Figure 6: Parallel Reordering Passes

\begin{tabular}{|l||r|r|r||}
\hline Benchmark & Rows & Cells & Nets \\
\hline fract & 6 & 149 & 163 \\
\hline struct & 21 & 1952 & 1920 \\
\hline primary1 & 17 & 833 & 904 \\
\hline primary2 & 22 & 3014 & 3029 \\
\hline biomed & 44 & 6514 & 7052 \\
\hline industry2 & 69 & 12637 & 13419 \\
\hline industry3 & 52 & 15433 & 21967 \\
\hline avqsmall & 79 & 21918 & 30038 \\
\hline avqlarge & 83 & 25178 & 33298 \\
\hline golem3 & 117 & 100312 & 217362 \\
\hline
\end{tabular}

Table 1: The placement benchmarks considered. The first four benchmarks are the ISCAS Benchmark circuits. Number of Rows determined using TimberWolf.

evenly among all processors using vector scatter-gather as shown in Figure 6. Because there is often a large number of rows in a placement, it is crucial to optimize memory reallocation; allocated memory is not released unless the space required to hold blocks of a new row is larger than the available space.

\section{Experiments}

The parallel implementation of the placement tool was evaluated for execution time and quality of solution on a cluster of eight $550 \mathrm{MHz}$ Pentium III workstations. The cluster is interconnected using a Myrinet network; the Myrinet LAN cards use a LANai $733 \mathrm{MHz}$ processor and 33MHz, 32-Bit PCI bus. The machines are also connected via switched 100Mbit/sec Ethernet. In [36], Feng Shui's performance was compared against commercially available tools and found to produce better results with smaller run times. In this paper, we focus on the performance of the parallel version. Unless otherwise indicated, all results are the average of five runs with different seeds. Table 1 shows the benchmarks used in the experiments. These benchmarks span a wide range of circuits, including the largest available public domain circuits.

Table 2 shows the execution times of the parallel algorithm (using scatter/gather). These results were about $30 \%$ faster than our initial implementation with round robin region distribution. The quality of the parallel version is not affected by the number of processors. The degradation in quality is due to the fact that the region processing is done in parallel and in phases. This is in contrast to the sequential version where the regions are updated sequentially, with every subsequent region using the updated values of the regions processed before it. We note that the smallest model (fract) suffers a slowdown in the parallel implementation; its size makes the communication overhead dominate the execution time. Note that even though the table shows the results for 2,4 and 8 processors only, the algorithm is not restricted to power of two processor configurations.

The results use a reorder window size of 6 . As we will show later, a window size of 6 was found to provide the best quality to execution time point (bigger window size took much longer to execute and provided marginal improvement). The drop in quality was small for most benchmarks (lower than 6\%). The exception was avqlarge and avqsmall which suffered around $10 \%$ reduction in solution quality. These two benchmarks also did not achieve good speedup despite their large size. We are currently looking more closely at their behavior to try to gain insight into their behavior; other studies report difficulties with these benchmarks [21]. The reduction in quality is significantly smaller than that reported in other parallel placement studies. For example, in one study the quality degradation of the parallel solution reached $30 \%$ (with an average of 14\%) [21] on a 4 processor study. In another study, the quality drop also reached over $30 \%$ with an average of around $20 \%$. Both studies only report results on 


\begin{tabular}{|l||r|r|r|r|r|r||}
\hline \multirow{2}{*}{ Benchmark } & \multicolumn{2}{|c|}{ Wire Length } & \multicolumn{4}{c||}{ Run Time (sec) } \\
\cline { 2 - 7 } & Sequential & Parallel (ratio) & Sequential & 2 Processors & 4 Processors & 8 Processors \\
\hline fract & 66242 & $69498.4(1.05)$ & 2.6 & 7 & 7 & 6.9 \\
\hline struct & 775636 & $797647.6(1.03)$ & 41.9 & 30.5 & 21.5 & 16.6 \\
\hline primary1 & 1053258 & $1064441(1.01)$ & 18.6 & 16.9 & 13.2 & 11.8 \\
\hline primary2 & 3747715 & $3855615.4(1.03)$ & 80.2 & 57.1 & 39.2 & 30.1 \\
\hline biomed & 3382200 & $3514514(1.04)$ & 162 & 111.8 & 79.4 & 64.5 \\
\hline industry2 & 15629154 & $16508315(1.06)$ & 359.4 & 227.2 & 155.5 & 117.8 \\
\hline industry3 & 45088174 & $47757192.8(1.06)$ & 538.6 & 338.5 & 218.1 & 157 \\
\hline avqsmall & 6041243 & $6626165.6(1.1)$ & 925.1 & 746.8 & 598.9 & 566.3 \\
\hline avqlarge & 6344469 & $7053757.8(1.11)$ & 1006 & 788.2 & 645.5 & 603.9 \\
\hline golem3 & 88959019 & $92830917(1.04)$ & 3235.9 & 2191.2 & 1243.1 & 918.1 \\
\hline
\end{tabular}

Table 2: Wire Lengths and Run Times for the Parallel Implementation Using Scatter/Gather on Myrinet

\begin{tabular}{|l||r|r|r|r||}
\hline \multicolumn{1}{|c||}{ Benchmark } & \multicolumn{4}{c||}{ Run Time (sec) } \\
\cline { 2 - 5 } & Sequential & 2 Proc. & 4 Proc. & 8 Proc. \\
\hline industry2 & 359.4 & 283 & 229.7 & $212.6(1.69)$ \\
\hline industry3 & 538.6 & 402.9 & 309.1 & $263.9(2.04)$ \\
\hline avqsmall & 925.1 & 894.6 & 816 & $772.9(1.20)$ \\
\hline avqlarge & 1006 & 946.2 & 872.6 & $842.3(1.19)$ \\
\hline golem3 & 3235.9 & 2328 & 1983 & 1635 \\
\hline
\end{tabular}

Table 3: Wire Lengths and Run Times for the Parallel Implementation Using Scatter/Gather on Ethernet

\begin{tabular}{|l||r|r|r||}
\hline \multirow{2}{*}{ Benchmark } & \multicolumn{2}{c|}{ Wire Length } & Improvement (\%) \\
\cline { 2 - 3 } & Without ID & With ID & \\
\hline fract & 68502 & 69498.4 & -1.4 \\
\hline struct & 804123 & 797647.6 & 0.8 \\
\hline primary1 & 1084639 & 1064441 & 1.9 \\
\hline primary2 & 3914142 & 3855615.4 & 1.5 \\
\hline biomed & 3506130 & 3514514 & -0.2 \\
\hline industry2 & 16488752 & 16508315 & -0.1 \\
\hline industry3 & 48973501 & 47757192.8 & 2.5 \\
\hline avqsmall & 6479309 & 6626165.6 & -2.3 \\
\hline avqlarge & 6947883 & 7053757.8 & -1.5 \\
\hline golem3 & 96020944 & 92830917 & 3.3 \\
\hline
\end{tabular}

a subset of the benchmarks used in this study.

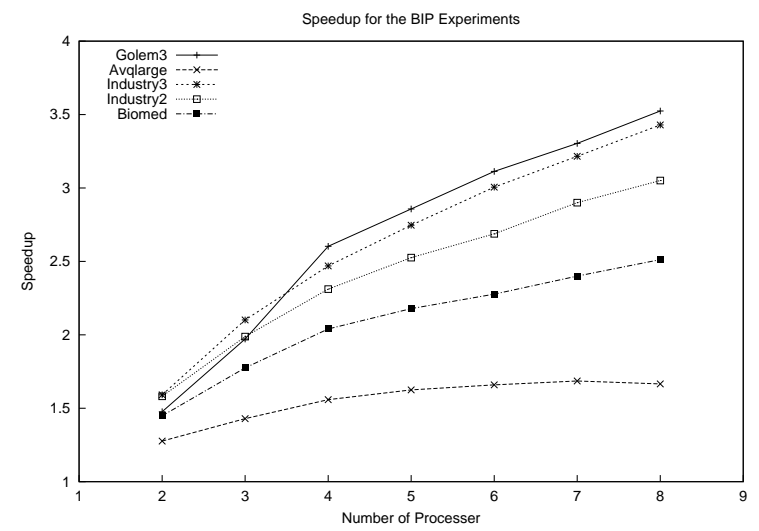

Figure 7: Speedup for the Myrinet/BIP Experiments

Table 4: Effect of Iterative Deletion

more clearly be seen in Figure 8 .

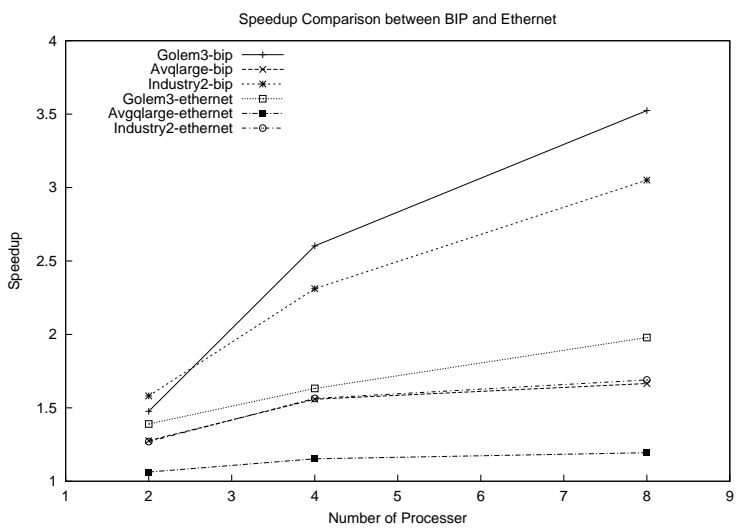

Figure 7 shows the speedup obtained by the parallel version on selected benchmarks. We note that the speedup generally increases with the size of the model; the larger the model the larger the granularity of the computation. The only exception is avqlarge - despite being the second largest model, it benefits least of the 5 models shown.

Table 3 shows the run times for selected benchmarks using ethernet communication. The quality results were identical to the Myrinet version. Clearly, the performance is significantly worse than the Myrinet version. This can

Figure 8: Speedup Comparison Myrinet/BIP vs. Ethernet

Iterative deletion proved useful in the sequential version of the tool, yielding a few percent improvement on average with a small increase in execution time. We attempted to verify whether iterative deletion will have the same effect on the parallel version. Table 4 presents the results of this study. While iterative deletion resulted in $0.7 \%$ improvement on average, it did perform worse for a 
number of benchmarks. Most notably, the two problematic benchmarks in terms of speedup and quality degradation (avqsmall and avqlarge) suffered the biggest degradation from using iterative deletion. Without those two, the improvement becomes $1.4 \%$.

We also investigated the effect of the size of the reorder window (from the default 6 to 8 ). The execution time rose sharply for all benchmarks, but the efficiency of the parallelism for the reorder portion of the algorithm raised the overall speedup (as can be seen in Table 5). We note however that the gain in quality is marginal; we would be better off to execute with window size 6 sequentially rather than window size 8 - the gain from the higher window size is smaller than the loss due to parallelism.

These results represent what is very much a work-inprogress. We expect to further refine the existing implementation in the following ways. Currently, the region list is relayed back to the master at the end of every partitioning step to update the dependencies, before resending the results back out to the slaves. This causes a lot of redundant communication as well as a sequential walk of the list. The next step is to allow a parallel walk of the list and update of the regions by doing an all to all exchange, instead of the "reduction" back to the master process. From the quality perspective, we are working on maintaining the sequential dependencies using a "wavefront pipeline" scheme. This formulation should produce the same quality as the sequential version, although the speedup will not be as high as the current formulation.

\section{Concluding Remarks}

With the exponential growth in the size of circuits under fabrication, efficient physical design tools are needed. Parallel processing offers the promise of increasing performance and capacity. The emergence of clusters as costeffective scalable high-performance computing platform brings the long overdue promise of parallel processing to the mainstream. The investigation of parallelization of physical design tools using clusters is timely.

In this paper, we presented experiences with parallelizing a state-of-the-art placement tool on a cluster of workstations. The sequential tool provides wire lengths comparable to those of a well known commercial tool, and results reported for the tool Capo indicate that placements are not difficult to route. The sequential implementation is efficient; the growth in run times is nearly linear with the size of the circuit. The tool uses a novel iterative deletion approach to allow the consideration of global objectives from within a traditional top-down placement framework. For more details please refer to the following paper [36].

Contrary to other efforts at parallel placement, we were able to obtain significant improvement in performance with minimal degradation in the quality of the layout. The degradation in the quality of the solution does not increase with the degree of parallelism. We explored several algorithmic and system optimizations and evaluated the implementation on a myrinet as well as a switched Ethernet network. We are still in the process of optimizing the implementation and are hopeful of achieving higher speedup in time for the final version of this paper.

We expect to continue to refine this tool both from an implementation and functionality perspectives. For example, timing driven placement is a significant concern for modern design. We are currently working with an industry research group to evaluate the performance of our approach on large designs under realistic delay rules. We note that delay optimization is perhaps more of a global phenomena than wire length minimization: meeting timing objectives may require modifications in many areas of a placement, and reductions in delay for some nets may require increased delay in others.

\section{References}

[1] T. Anderson, D. Culler, and D. Patterson. The case for NOW (network of workstations). IEEE Micro, 15(1), Feb. 1995.

[2] P. Banerjee. Parallel Algorithms for VLSI Computer-Aided Design. Prentice Hall, Englewood Cliffs, New Jersey, 1994. (Chapter 3).

[3] D. Becker, T. Sterling, D. Savarese, J. Dorband, U. Ranawak, and C. Packer. BEOWULF: A parallel workstation for scientific computation. In International Conference on Parallel Processing, 1995.

[4] M. Blumrich, R. Alpert, Y. Chen, D. Clark, S. Damianakis, C. Dubnicki, E. Felten, L. Iftode, K. Li, M. Martonosi, and R. Shillner. Design choices in the shrimp system: An empirical study. In Proceedings of the $25^{\text {th }}$ Annual ACM/IEEE International Symposium on Computer Architecture, June 1998.

[5] N. Boden, D. Cohen, and W. Su. Myrinet: A gigabit-persecond local area network. IEEE Micro, 15(1), Feb. 1995.

[6] M. A. Breuer. A class of min-cut placement algorithms. In Proc. Design Automation Conf, pages 284-290, 1997.

[7] A. E. Caldwell, A. B. Kahng, and I. L. Markov. Optimal partitioners and end-case placers for standard cell layout. In Proc. Int. Symp. on Physical Design, pages 90-96, 1999.

[8] F. Darema, S. Kirkpatrick, and V. Norton. Parallel algorithms for chip placement by simulated annealing. IBM Journal of Research and Development, May 1987.

[9] C. Dubnicki, A. Bilas, Y. Chen, S. Damianakis, and K. Li. VMMC-2: Efficient support for reliable, connectionoriented communication. In Hot Interconnects $V$, Aug. 1997.

[10] A. E. Dunlop and B. W. Kernighan. A procedure for placement of standard-cell VLSI circuits. IEEE Trans. 


\begin{tabular}{|l||r|r|r|r|r|r||}
\hline \multicolumn{1}{|c||}{ Benchmark } & \multicolumn{7}{|c||}{ Window Size } \\
\cline { 2 - 7 } & & \multicolumn{3}{c||}{6} & \multicolumn{3}{c||}{8} \\
\cline { 2 - 7 } & Time & Speedup & Wire Length & Time & Speedup & Wire Length \\
\hline struct & 16.7 & 2.5 & 789777 & 34.3 & 3.32 & 782683 \\
\hline primary2 & 30.2 & 2.65 & 3863183 & 77.8 & 3.69 & 3852182 \\
\hline biomed & 64.3 & 2.52 & 3494020 & 110 & 3.22 & 3472616 \\
\hline industry2 & 117.9 & 3.04 & 16444527 & 301.1 & 4.1 & 16380765 \\
\hline avqsmall & 561.1 & 1.64 & 6670242 & 749.7 & 2.88 & 6603005 \\
\hline
\end{tabular}

Table 5: Effect of Reording Window Size - 8 Processors

on Computer-Aided Design of Integrated Circuits andSystems, CAD-4(1):92-98, Jan. 1985.

[11] H. Eisenmann and F. M. Johannes. Generic global placement and floorplanning. In Proc. Design Automation Conf, pages 269-274, 1998.

[12] C. M. Fiduccia and R. M. Mattheyses. A linear-time heuristic for improving network partitions. In Proceedings of the $19^{\text {th }}$ IEEE Design Automation Conference, pages 175-181, 1982.

[13] P. Geoffray, L. Prylli, and B. Tourancheau. BIP-SMP: High performance message passing over a cluster of commodity SMPs. In Proceedings of Supercomputing (SC99), Nov. 1999.

[14] D. J.-H. Huang and A. B. Kahng. Partitioning based standard cell global placement with an exact objective. In Proc. Int. Symp. on Physical Design, pages 18-25, 1997.

[15] M. Ibel, K. Schauser, C. Scheiman, and M. Weis. High performance cluster computing using SCI. In Hot Interconnects V, Aug. 1997.

[16] R. Jayaraman and R. Rutenbar. Floorplanning by annealing on a hypercube multiprocessor. In Proceedings of the International Conference on Computer-Aided Design, pages 346-349, Nov. 1987.

[17] G. Karypis, R. Aggarwal, V. Kumar, and S. Shekhar. Multilevel hypergraph partitioning: Application in VLSI domain. In Proc. Design Automation Conf, pages 526-529, 1997.

[18] B. W. Kernighan and S. Lin. An efficient heuristic procedure for partitioning graphs. Bell System Technical Journal, 49:291-307, 1970.

[19] S. Kim, B. Ramkumar, J. Chandy, S. Parkes, and P. Banerjee. ProperPLACE: a portable parallel algorithm for standard cell placement. In Proceedings of the 8th International Parallel Processing Symposium (IPPS'94), Apr. 1994.

[20] R. Kling and P. Banerjee. Concurrent ESP: a placement algorithm for execution on distributed processors. In Proceedings of the International Conference on ComputerAided Design, pages 354-357, Nov. 1987.

[21] T. Koide, M. Ono, S. Wakabayashi, and Y. Nishimaru. ParPOPINS: a timing-driven parallel placement method with the elmore delay model for row based VLSIs. In Proceedings of the Design Automation Conference (DAC'97), 1997.

[22] S. Kravitz and R. Rutenbar. Placement by simulated annealing on a multiprocessor. IEEE Transactions on Computer Aided Design of Integrated Circuits and Systems, 6(4):534-549, June 1987.
[23] P. H. Madden. Partitioning by iterative deletion. In Proc. Int. Symp. on Physical Design, pages 83-89, 1999.

[24] S. Mohan and P. Mazumder. Wolverines: Standard cell placement on a network of workstations. IEEE Transactions on Computer Aided Design of Integrated Circuits and Systems, 12(9):1312-1326, Sept. 1993.

[25] M-VIA: Virtual interface architecture for linux, 2001. http://www.nersc.gov/research/FTG/via/.

[26] S. Pakin, M. Lauria, and A. Chien. High performance messaging on workstations: Illinois Fast Messages (FM) for Myrinet. In Proceedings of Supercomputing (SC'95), 1995.

[27] G. Pfister. In Search of Clusters, 2nd Ed. Prentice Hall, 1998.

[28] L. Prylli. BIP messages user manual, 1998. Available at http://lhpca.univ-lyon1.fr/BIP-manual/ index.html.

[29] L. A. Sanchis. Multiple-way network partitioning with different cost functions. IEEE Trans. on Computers, 42(22): 1500-1504, 1993.

[30] Scalable Computing Lab. SCL cluster cookbook: Building your own clusters for parallel computation, 1998. http://www.scl.ameslab.gov/ Projects/ClusterCookbook.

[31] K. Shahookar and P. Mazumder. Vlsi cell placement techniques. ACM Computing Surveys, 23(2):143-220, June 1991.

[32] P. R. Suaris and G. Kedem. An algorithm for quadrisection and its application to standard cell placement. IEEE Trans. on Circuits and Systems, 35(3):394-303, 1988.

[33] W.-J. Sun and C. Sechen. Efficient and effective placement for very large circuits. IEEE Trans. on Computer-Aided Design of Integrated Circuits andSystems, 14(3):349-359, 1995.

[34] Virtual interface architecture (VIA) specification, 2001. http: / / www. viarch.org.

[35] M. Welsh, A. Basu, and T. von Eicken. Atm and fast ethernet network interfaces for user-level communication. In Proceedings of the Third High-Performance Computer Architecture Conference (HPCA'97), Feb. 1997.

[36] M. Yeldiz and P. H. Madden. Global objectives for standard cell placement. In Design Automation Conference (DAC’2000), 2000. 